\title{
Vertical Spatial Sensitivity and Exploration Depth of Low-Induction-Number Electromagnetic-Induction Instruments
}

\author{
James B. Callegary, * Ty P. A. Ferré, and R. W. Groom
}

\begin{abstract}
Vertical spatial sensitivity and effective depth of exploration $\left(d_{\mathrm{e}}\right)$ of low-induction-number (LIN) instruments over a layered soil were evaluated using a complete numerical solution to Maxwell's equations. Previous studies using approximate mathematical solutions predicted a vertical spatial sensitivity for instruments operating under LIN conditions that, for a given transmitter-receiver coil separation (s), coil orientation, and transmitter frequency, should depend solely on depth below the land surface. When not operating under LIN conditions, vertical spatial sensitivity and $d_{\mathrm{e}}$ also depend on apparent soil electrical conductivity $\left(\sigma_{a}\right)$ and therefore the induction number $(\beta)$. In this new evaluation, we determined the range of $\sigma_{a}$ and $\beta$ values for which the LIN conditions hold and how $d_{\mathrm{e}}$ changes when they do not. Two-layer soil models were simulated with both horizontal (HCP) and vertical (VCP) coplanar coil orientations. Soil layers were given electrical conductivity values ranging from 0.1 to $200 \mathrm{mS} \mathrm{m}$. As expected, $d_{e}$ decreased as $\sigma_{a}$ increased. Only the least electrically conductive soil produced the $d_{\mathrm{e}}$ expected when operating under LIN conditions. For the VCP orientation, this was $1.6 s$, decreasing to $0.8 \mathrm{~s}$ in the most electrically conductive soil. For the HCP orientation, $d_{\mathrm{e}}$ decreased from $0.76 \mathrm{~s}$ to 0.51s. Differences between this and previous studies are attributed to inadequate representation of skin-depth effect and scattering at interfaces between layers. When using LIN instruments to identify depth to water tables, interfaces between soil layers, and variations in salt or moisture content, it is important to consider the dependence of $d_{\mathrm{e}}$ on $\sigma_{\mathrm{a}}$.
\end{abstract}

$\mathrm{L}$ OW-INDUCTION-NUMBER frequency-domain electromagnetic-induction instruments use the propagation of alternating electromagnetic fields through the soil to measure the apparent soil electrical conductivity $\left(\sigma_{\mathrm{a}}, \mathrm{mS} \mathrm{m}^{-1}\right)$. This measured property is a complicated average of spatially distributed localized electrical conductivities in the subsurface. Apparent soil electrical conductivity is affected by several factors including water content, mineralogy, temperature, soil texture, porosity, permeability, and salinity. Instruments capable of operating as LIN FEM instruments include the EM38, EM31, and EM34 (Geonics Ltd., Mississauga, ON), the DUALEM instruments series (DUALEM, Inc., Milton, ON), and the GEM instrument series (Geophex Ltd., Raleigh, NC). The range of applications of LIN FEM instruments for environmental and hydrologic characterization and monitoring is large and increasing. Applications include aquifer extent and water

J.B. Callegary, U.S. Geological Survey, 520 N. Park Ave., Tucson, AZ 85719; T.P.A. Ferré, Hydrology and Water Resources, Univ. of Arizona, Tucson, AZ 85721; R.W. Groom, PetRos EiKon, Inc., 222 Snidercroft Rd., Concord, ON L4K 2K1, Canada. Any use of trade, product, or firm names in this publication is for descriptive purposes only and does not imply endorsement by the U.S. government. Received 25 Aug. 2006. *Corresponding author (jcallega@usgs.gov).

Published in Vadose Zone Journal 6:158-167 (2007).

Original Research

doi:10.2136/vzj2006.0120

(c) Soil Science Society of America

677 S. Segoe Rd., Madison, WI 53711 USA content studies (António and Pacheco, 2002; Schneider and Kruse, 2003; Sheets and Hendrickx, 1995), and lithology, soil salinity, and soil texture mapping (Benjoudi et al., 2002; Lesch et al., 1998; Paine, 2003; Stroh et al., 2001; Sudduth et al., 2005; Triantafilis et al., 2005; Yoder et al., 2001). The LIN FEM instruments also have been used to delineate landfills (Lanz et al., 1998; Nyquist and Blair, 1991), contaminant plumes (Matias et al., 1994), and areas of active recharge (Salama et al., 1994).

Quantitative applications of LIN FEM instruments to hydrologic investigations depend on the ability to transform measured electrical conductivities into vertical and horizontal variations of hydrologically relevant properties. The accuracy of these transformations relies, primarily, on (i) calibrating electrical conductivity to a property of interest (e.g., volumetric water content) either through qualitative or numerical inversion, and (ii) mapping interpreted hydrologic variables based on measurement locations. A comprehensive review of modeling and inversion as applied to electric and electromagnetic methods was recently published by Pellerin and Wannamaker (2005). In our investigation, we exclusively examined the latter, less commonly considered issue. Specifically, we used forward numerical models of layered soils to study the effect of variations in $\sigma_{\mathrm{a}}$ on the vertical spatial sensitivity of LIN FEM instruments. This information is useful when attempting to assign depths to properties such as soil water content (e.g., depth to the water table), soil texture, salinity, and hydraulic conductivity.

It is commonly assumed that, under most conditions, the sample depth (or sample volume in three dimensions) of LIN FEM instruments is independent of the subsurface electrical conductivity. McNeill (1980) defined "effective depths of exploration" $\left(d_{\mathrm{e}}\right)$ based on the vertical spatial sensitivity of LIN FEM instruments in homogeneous and horizontally layered soils, which he computed using an asymptotic approximation of Maxwell's equations. The asymptotic approximation is based on the assumption that the induction number $(\beta)$ is very small. (As such, this solution is sometimes referred to as the "LIN approximation," and it formed the basis for development of many LIN FEM instruments.) The induction number is the ratio of the intercoil separation $(s)$ (Fig. 1) to the skin depth (ס) (Kaufman and Keller, 1983; Spies, 1989):

$$
\beta=\frac{s}{\delta}=\frac{s}{\sqrt{\frac{2}{\sigma_{\mathrm{a}} \omega \mu}}}
$$

where $\omega$ is angular frequency $\left(\mathrm{s}^{-1}\right)$, and $\mu$ is magnetic permeability $\left(\mathrm{H} \mathrm{m}^{-1}\right)$, normally assumed to be constant

Abbreviations: FEM, frequency-domain electromagnetic-induction; HCP, horizontal coplanar; LIN, low-induction-number; VCP, vertical coplanar. 


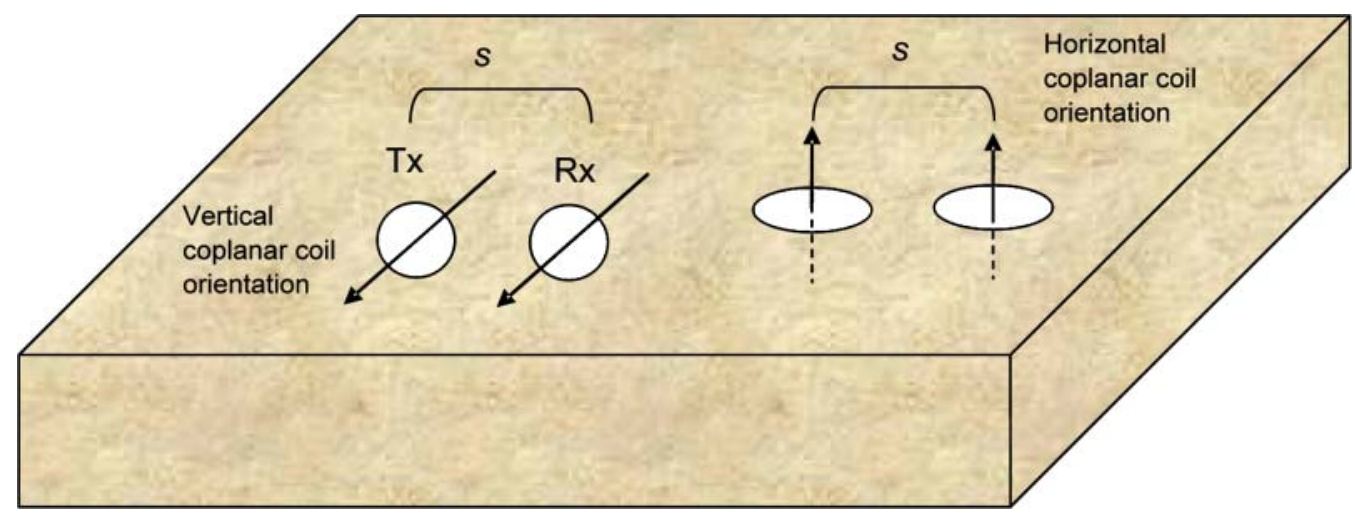

Fig. 1. Low-induction-number frequency-domain electromagnetic-induction instruments, two coils with an intercoil separation (s). One coil transmits $(T x)$ the primary magnetic field and one coil receives $(R x)$ a combination of the primary and secondary fields. Coils may be oriented either horizontally or vertically with respect to the ground surface as indicated by directional arrows.

and equivalent to its free-space value $\left(\mu_{0}\right)$. Skin depth is the depth at which the transmitted magnetic field strength has decayed to $\mathrm{e}^{-1}$ of its initial magnitude at a reference point. In the low-frequency limit, $\delta$ varies inversely with the square root of $\sigma_{\mathrm{a}}$. As $\sigma_{\mathrm{a}}$ increases, $\delta$ decreases and $\beta$ increases, effectively decreasing instrument sensitivity with increasing depth, and decreasing $d_{\mathrm{e}}$. Some researchers used the LIN approximation in their investigations, but did not state the range of $\beta$ required for a valid approximation. McNeill (1980) asserted that the LIN approximation holds where $\beta$ is $<<1$ and recommended that LIN FEM instruments be used in environments where $\sigma_{\mathrm{a}} \leq 100 \mathrm{mS} \mathrm{m}^{-1}$ (which for $\omega=9800 \mathrm{~Hz}$ and $s=3.66 \mathrm{~m}$, corresponds to $\beta \leq 0.23$ ). Wait (1962) defined this as $\beta$ less than about 0.3 and Frischknecht (1987) defined the criterion as $\beta<$ 0.02 . This disagreement makes it unclear under what values of $\beta$ the LIN approximation can be applied.

In this study, we used a standard commercial forward numerical code of electromagnetic field propagation (EMIGMA, PetRos EiKon, Inc., 2004) to examine the $d_{\mathrm{e}}$ of FEM instruments. A numerical code does not require the LIN approximation, and is therefore more accurate in its representation of physical processes affecting electromagnetic wave propagation and $d_{\mathrm{e}}$ of FEM instruments. A numerical code also allows for identifying conditions under which the LIN approximation might not be applicable. Specifically, like McNeill (1980), we defined the vertical spatial sensitivity of an FEM instrument in horizontally layered soil. Additionally, we varied $\sigma_{\mathrm{a}}$ to determine at what $\beta$ values the LIN approximation holds and when it does not, to determine the effect on $d_{\mathrm{e}}$. Based on our findings, we redefined the conditions under which the LIN approximation should be used.

\section{THEORY}

Low-induction-number FEM instruments operate with a transmitter coil to generate an alternating magnetic field $(\partial \mathbf{H} / \partial t)$ where $\mathbf{H}$ is the vector magnetic field strength $\left(\mathrm{A} \mathrm{m}^{-1}\right)$ and $t$ is time (s). This magnetic field propagates into the subsurface, where it induces alternating currents as described by Faraday's law:

$$
\nabla \times \mathbf{E}=-\mu \frac{\partial \mathbf{H}}{\partial t}
$$

where $\mathbf{E}$ is the vector electric field strength $\left(\mathrm{V} \mathrm{m}^{-1}\right)$. This equation states that a time-varying magnetic field will produce an electric field whose curl is equal to the negative of the time derivative of the magnetic field. The electric field induces currents in the soil, which, in turn, produce their own magnetic fields whose polarity and magnitude oppose the change in the incident field. The incident magnetic field often is called the primary field and the field generated by the induced currents is called the secondary field. These two fields combine to induce currents in the receiver coil in accordance with Faraday's law. The transmitter and receiver coils, and associated magnetic dipoles, can be oriented relative to each other and to the soil surface. Orientations considered in this study were horizontal coplanar (both coils lie flat on the ground) and vertical coplanar (coils are upright and coplanar) (Fig. 1).

Maxwell's equations generally describe the macroscopic behavior of electromagnetic fields (GomezTreviño et al., 2002). Numerical as well as analytical and approximate solutions to these equations can be used to investigate the propagation of subsurface electromagnetic fields under specific conditions. Typically, analytical solutions can be derived only for simple scenarios such as uniform half-spaces (Ward and Hohmann, 1988). Even with geometric simplifications, additional restrictive simplifying assumptions are required to solve the equations analytically. Approximate solutions, such as the solution used by McNeill (1980), use restrictive physical and mathematical assumptions to arrive at solutions that are approximately valid in some limited physical regime. Numerical codes developed in the 1980s (Anderson, 1984; Tabbagh, 1985; Wannamaker et al., 1984) allow consideration of more complex, realistic soil models. In the development of EMIGMA, the code used for this study, an attempt was made not to simplify the mathematics in any way. Although small numerical errors still result from the calculations, errors in EMIGMA are generally less than errors expected from instruments or geologic noise. In addition to allowing more general geometries, numerical codes can more accurately represent processes such as skin depth effects and scattering. Scattering, which includes reflection, refraction, current channeling, and induction, describes the distortion of 
electromagnetic waves at spatial discontinuities in electrical and magnetic properties of soil. These various processes affect propagation of the incident electromagnetic field and consequently the propagation and magnitude of secondary electromagnetic fields.

McNeill (1980) discussed part of the theory and assumptions of the mathematical approximations that provide the basis for development, use, and interpretation of LIN FEM instruments. The derivation of equations discussed by McNeill (1980) was developed in greater detail by Belluigi (1949), Wait (1955, 1962), Kaufman and Keller (1983), and Gomez-Treviño et al. (2002). The LIN approximation is derived from Maxwell's equations for a one-dimensional soil (homogeneous, layered, or arbitrary electrical conductivity $[\sigma]$ variations with depth) in which transmitter frequency is low and $s$ (Fig. 1) is small compared with $\delta(\mathrm{m})$ of magnetic fields in the soil (LIN conditions). For these conditions, vertical spatial sensitivity and $d_{\mathrm{e}}$ are independent of $\sigma_{\mathrm{a}}$ and the LIN approximation is valid. Opinions on what constitutes LIN conditions, however, can vary by investigator. For an EM31 instrument with a coil spacing of $3.66 \mathrm{~m}$ and an operating frequency of $9800 \mathrm{~Hz}$, the LIN assumptions might be valid for $\sigma_{\mathrm{a}}<$ $200 \mathrm{mS} \mathrm{m}^{-1}$ for Wait's (1962) criterion, $\leq 100 \mathrm{mS} \mathrm{m}^{-1}$ for McNeill (1980), or $<0.8 \mathrm{mS} \mathrm{m}{ }^{-1}$ for Frischknecht (1987). Table 1 shows the corresponding values for resistivity and skin depth. A practical difference exists between $0.8 \mathrm{mS} \mathrm{m}^{-1}$ and the two higher values. Electrical conductivity of most surficial material in the continental USA probably lies between 0.8 and $100 \mathrm{mS} \mathrm{m}^{-1}$ (Keller and Frischknecht, 1966). If either McNeill's or Wait's criterion is correct, then the LIN approximation and McNeill's associated guides to data interpretation should be able to be used without significant problems. If Frischknecht's is the correct criterion, however, soil and rock electrical conductivities at most field sites probably are too high for the LIN approximation to be valid. As a result, inferences of true $\sigma_{\mathrm{a}}$, and thicknesses and numbers of soil layers based on these assumptions, could be incorrect.

Table 1. Induction number calculated for electrical conductivities with low-induction-number (LIN) condition criteria proposed by several researchers at frequency $=9800 \mathrm{~Hz}$, coil spacing $=3.66 \mathrm{~m}$, and magnetic permeability of free space $=4 \pi \times 10^{-7} \mathrm{H} \mathrm{m}^{-1}$.

\begin{tabular}{|c|c|c|c|c|}
\hline $\begin{array}{l}\text { Apparent } \\
\text { soil electrical } \\
\text { conductivity, } \sigma_{\mathbf{a}}\end{array}$ & $\begin{array}{l}\text { Resistivity, } \\
\sigma^{-1}\end{array}$ & $\begin{array}{c}\text { Skin } \\
\text { depth, } \delta\end{array}$ & $\begin{array}{c}\text { Induction } \\
\text { no., } \boldsymbol{\beta}\end{array}$ & $\begin{array}{c}\text { LIN condition } \\
\text { criterion }(\beta \leq x) \\
\text { proposed by }\end{array}$ \\
\hline $\mathbf{m S ~ m} \mathbf{m}^{-1}$ & $\omega \mathbf{m}$ & m & & \\
\hline 10000 & 0.1 & 2 & 2.3 & $\mathbf{n} / \mathbf{a}$ \\
\hline 1000 & 1 & 5 & 0.72 & $\mathbf{n} / \mathbf{a}$ \\
\hline 500 & 2 & 7 & 0.51 & $\mathbf{n} / \mathbf{a}$ \\
\hline 200 & 5 & 11 & 0.32 & Wait (1962) \\
\hline 100 & 10 & 16 & 0.23 & McNeill (1980) \\
\hline 50 & 20 & 23 & 0.16 & $\mathbf{n} / \mathbf{a}$ \\
\hline 20 & $\mathbf{5 0}$ & 36 & 0.10 & $\mathbf{n} / \mathbf{a}$ \\
\hline 10 & 100 & 51 & 0.072 & $\mathbf{n} / \mathbf{a}$ \\
\hline 5 & 200 & 72 & 0.051 & $\mathbf{n} / \mathbf{a}$ \\
\hline 2 & 500 & 114 & 0.032 & $\mathbf{n} / \mathbf{a}$ \\
\hline 0.8 & 1300 & 183 & 0.020 & Frischknecht (1987) \\
\hline 0.2 & 5000 & 359 & 0.010 & $\mathbf{n} / \mathbf{a}$ \\
\hline 0.1 & 10000 & 508 & 0.007 & $\mathbf{n} / \mathbf{a}$ \\
\hline 0.01 & 100000 & 1608 & 0.002 & $\mathbf{n} / \mathbf{a}$ \\
\hline
\end{tabular}

When site characteristics and instrument parameters combine to satisfy the LIN conditions, the quadrature component of the secondary magnetic field strength, $H_{\mathrm{s}}$, is linearly dependent on $\sigma_{\mathrm{a}}$ (McNeill, 1980). The quadrature component of the secondary magnetic field is that part of the secondary magnetic field that is $90^{\circ}$ out of phase with the primary field (Telford et al., 1990):

$$
\sigma_{\mathrm{a}}=\frac{4}{\omega \mu_{0} s^{2}} \frac{\left(H_{\mathrm{s}}\right)_{\text {QuadratureComponent }}}{H_{\mathrm{p}}}
$$

where $H_{\mathrm{p}}$ is the primary magnetic field strength. Coil orientation, $s$, and $\omega$ usually are fixed for a given measurement of $\sigma_{a}$; however, if $s$ is increased or $\omega$ decreased, $d_{\mathrm{e}}$ increases. The effective depth of exploration will also increase by changing the magnetic dipole orientation from HCP to VCP, because VCP sensitivity distribution peaks at greater depths than the HCP distribution (Fig. 2).

McNeill (1980) provided a simple form of spatial sensitivity analysis using his "cumulative response," which we change to "cumulative sensitivity," (a measure of an instrument's distribution of sensitivity in the subsurface). Cumulative sensitivity (CS) can be used to determine the sensitivity of LIN FEM instruments to all material above or below a given depth. Depths are normalized to facilitate comparisons of instruments with different intercoil separations. The normalized depth, $z$, is the actual depth divided by the intercoil separation, $s$, and is referred to in terms of $s$. For instance, a 7.32-m depth for an instrument with a 3.66-m intercoil separation is referred to as $2 s$. Equations of cumulative sensitivity for the horizontal $\left(\mathrm{CS}_{\mathrm{HCP}}\right)$ and vertical $\left(\mathrm{CS}_{\mathrm{VCP}}\right)$ coil orientations as a function of normalized depth (McNeill, 1980) are

$$
\begin{gathered}
\mathrm{CS}_{\mathrm{HCP}}(z)=\int_{z}^{\infty} \operatorname{LS}_{\mathrm{HCP}}(z) \mathrm{d} z=\frac{1}{\left(4 z^{2}+1\right)^{1 / 2}} \\
\mathrm{CS}_{\mathrm{VCP}}(z)=\int_{z}^{\infty} \operatorname{LS}_{\mathrm{VCP}}(z) \mathrm{d} z=\left(4 z^{2}+1\right)^{1 / 2}-2 z
\end{gathered}
$$

where local sensitivity (LS) is the relative contribution of material at a given depth to the measured value of $\sigma_{\mathrm{a}}$. A CS value represents the fraction of the secondary field at the receiver that originates between a depth $z$ and infinite depth. When $z$ is small and CS is near 1, most of the measured response comes from soil at a depth $>z$. The part of the response due to material in the interval between the surface $(z=0)$ and $z$ is $1-$ CS. By plotting the functions in Eq. [4] and [5] and locating the CS value associated with McNeill's exploration depths, it appears that McNeill used a CS value of 0.7 (or 70\%) to define $d_{\mathrm{e}}$ (McNeill, 1980, p. 5-6). For example, when CS $=0.3$ for $\mathrm{HCP}$ orientation, about $30 \%$ of the measured response is attributable to material at depths $>0.76 s$ (Fig. 2). Therefore, the interval between $0 s$ and $0.76 s$ contributes $70 \%$ of the HCP orientation response. Material located between $0 s$ and $1.6 s$ contributes $70 \%$ of the VCP orientation response (Fig. 2). These depths, $0.76 s$ and $1.6 s$, represent $d_{\mathrm{e}}$ for these orientations under LIN conditions.

In a two-layer soil for which CS is calculated from infinite depth to the contact between the layers, the top 


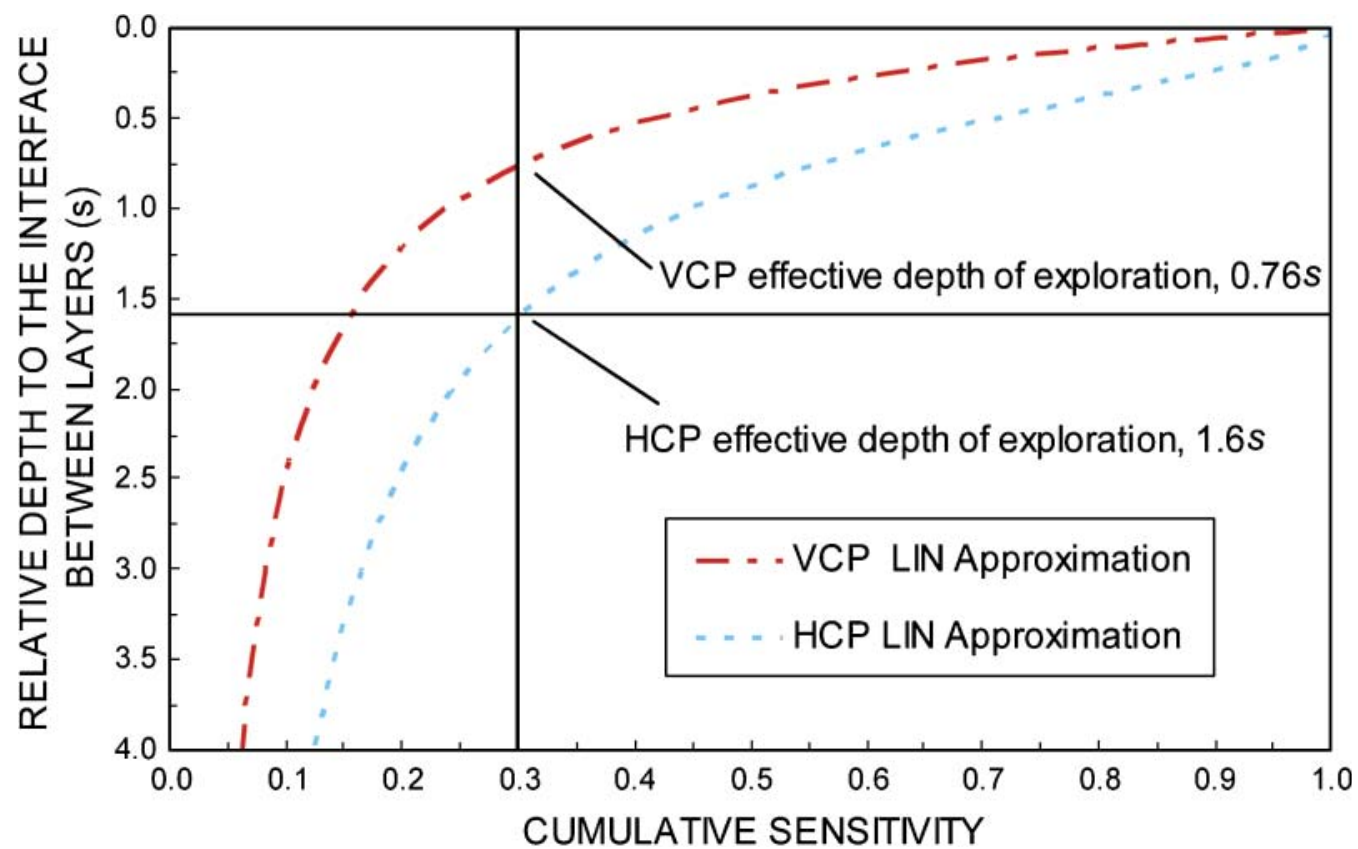

Fig. 2. Cumulative sensitivity and relative depth to interface between layers (McNeill, 1980). Approximations are for vertical (VCP) and horizontal (HCP) coplanar coil orientations. McNeill's "effective depth of exploration" is indicated by a cumulative sensitivity value of 0.3 . Cumulative sensitivity of low-induction-number (LIN) instruments is calculated from infinity to a given relative depth. Relative depth is depth divided by intercoil separation.

layer's contribution to the measured response is $1-\mathrm{CS}$. In such soil, CS near 1 represents a nearly homogeneous soil where $\sigma_{\mathrm{a}}$ is equal to $\sigma$ of the lower layer. Cumulative sensitivity near 0 represents a nearly homogeneous soil where $\sigma_{\mathrm{a}}$ is equal to $\sigma$ of the upper layer. In a two-layer soil, $\sigma_{\mathrm{a}}$ can be calculated by

$$
\sigma_{\mathrm{a}}=\sigma_{\text {Layer1 }}(1-\mathrm{CS})+\sigma_{\text {Layer2 }} \mathrm{CS}
$$

This calculation is equivalent to Eq. [5] from McNeill (1980). The value of CS can be determined based on $\sigma_{\mathrm{a}}$ of a two-layered soil if $\sigma$ values of each layer $\left(\sigma_{\text {Layer1 }}\right.$ and $\left.\sigma_{\text {Layer2 }}\right)$ are known. Specifically, rearranging Eq. [6] to solve for CS gives

$$
\mathrm{CS}=\frac{\sigma_{\mathrm{a}}-\sigma_{\text {Layer1 }}}{\sigma_{\text {Layer2 }}-\sigma_{\text {Layer1 }}}
$$

\section{METHODS}

In this study, the software EMIGMA (PetRos EiKon, Inc., 2004) was used to conduct forward numerical simulations of a Geonics EM31 instrument response over a nearly homogeneous or horizontally layered soil. The software was developed to calculate three-dimensional, electromagnetic-field propagation in complex environments for a great variety of instrument systems. The code, before commercial release, was calibrated against a number of academic and internal industry codes, as well as codes published in several articles and doctoral theses. An intercoil separation of $3.66 \mathrm{~m}$ and a transmitting frequency of $9800 \mathrm{~Hz}$ were used for all simulations. These are the instrument parameters used in the Geonics EM31 instrument. The software represents coils as alternating magnetic dipoles, which, for these simulations, were placed $0.05 \mathrm{~m}$ above the ground. Magnetic permeability was constant for all simulations and set equal to its free-space value $\left(\mu_{0}\right)$. The ratio of the secondary to the primary magnetic field mag- nitude (\%) was used as output format instead of $\sigma_{\mathrm{a}}$, because this is the more common output format of many instruments. Moreover, $\sigma_{\mathrm{a}}$ is inherently less accurate than the magnetic field ratio because it can only be calculated through the use of numerical inversion routines or approximate models.

All results are presented as CS for direct comparison with the results of LIN approximation. We simulated two-layer soils differing only in $\sigma$ of the layers. Individual-layer electrical conductivities ranged from 0.1 to $200 \mathrm{mS} \mathrm{m}^{-1}$. In each twolayered soil, upper and lower layer $\sigma$ varied from nearly equal (e.g., upper layer, $99 \mathrm{mS} \mathrm{m}^{-1}$; lower layer, $100 \mathrm{mS} \mathrm{m}^{-1}$ ), to very different values (e.g., upper layer, $100 \mathrm{mS} \mathrm{m}^{-1}$; lower layer, $0.1 \mathrm{mS} \mathrm{m}^{-1}$ ). For each $\sigma$ value used in the simulations, the response (ratio of magnetic field magnitudes) was determined for a homogeneous half-space for both horizontaland vertical-coplanar coil orientations. A series of simulations was then performed by varying upper layer thickness. Equation [7] was used to determine the numerical CS value from the simulation results. One value in the CS distribution was calculated from each simulation. Each CS value was plotted as a function of depth to the interface between layers to generate a curve for comparison with curves in Fig. 2. Comparing LIN-approximation curves facilitated determining the effects of changing individual-layer electrical conductivities on the vertical spatial sensitivity distribution.

Cumulative sensitivity was calculated for two soil-model types: Type 1 and Type 2 (Fig. 3). For a given series of simulations, a constant- $\sigma$ upper layer was used for Type 1 soil models and constant $-\sigma$ lower layer was used for Type 2 soil models. Each model type was evaluated at two ranges of $\sigma_{a}$, one electrically "resistive" and one electrically "conductive," in which the soil model was composed of two layers and the thickness of the top layer ranged from 0.001 to $18 \mathrm{~m}$ (or $0 s-4.9 s$ ). For all soil models, the bottom layer was essentially infinitely thick $\left(1 \times 10^{8} \mathrm{~m}\right)$. For Type 1 resistive soil models, $\sigma$ of the upper layer was held constant at $0.1 \mathrm{mS} \mathrm{m}^{-1}$ and $\sigma$ of the lower layer was given one of three values: $0.2,10$, or $100 \mathrm{mS} \mathrm{m}^{-1}$. For Type 2 resistive soil models, $\sigma$ of the upper layer was given values of $0.2,10$, or 

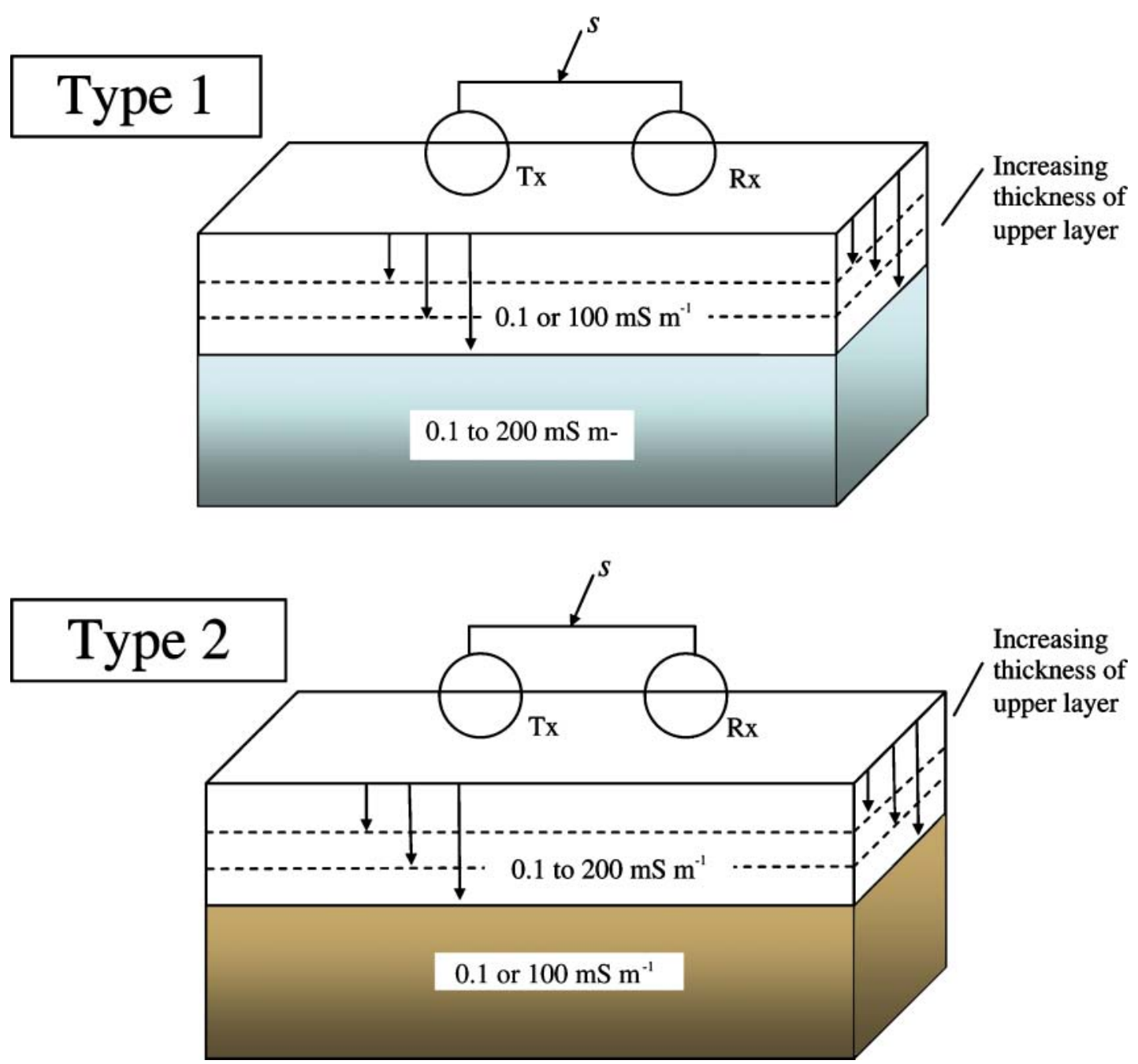

Fig. 3. Each set of cumulative-sensitivity simulations was based on a two-layer soil. Electrical conductivity of the upper layer in soil model Type 1 and the lower layer in soil model Type 2 were fixed at $0.1 \mathrm{mS} \mathrm{m}^{-1}$ for the electrically resistive case or $100 \mathrm{mS} \mathrm{m} \mathrm{m}^{-1}$ for the electrically conductive case. The variable electrical-conductivity layer was given values ranging from 0.1 to $200 \mathrm{mS} \mathrm{m} \mathrm{m}^{-1}$. For a given two-layer soil model in each set of simulations, the thickness of the upper layer increased from 0.001 to $18 \mathrm{~m}$. Tx indicates transmitter coil; Rx indicates receiver coil; and $s$ is the intercoil separation.

$100 \mathrm{mS} \mathrm{m}^{-1}$, and $\sigma$ of the lower layer was held constant at $0.1 \mathrm{mS} \mathrm{m}^{-1}$. These resistive soil models were used to match the LIN approximate solution and to study vertical sensitivity distribution changes in more electrically resistive environments. To mimic conditions common in near-surface investigations, a series of electrically conductive simulations were performed at higher $\sigma_{\mathrm{a}}$ values using the same Type 1 and 2 soil-model scenarios. Such conditions might be found at waste sites contaminated with inorganic compounds, at landfills, or in saturated clays. The constant- $\sigma$ layer was $100 \mathrm{mS} \mathrm{m}^{-1}$ and five soils were simulated in which the variable layer $\sigma$ ranged from 0.1 to $200 \mathrm{mS} \mathrm{m}^{-1}$.

\section{RESULTS AND DISCUSSION}

Cumulative sensitivity was plotted as a function of depth to the interface between upper and lower layers to compare the vertical sensitivity distribution of the LIN approximation over a layered soil with those derived from numerical simulations (Fig. 4-6). Except for two soils with a $200 \mathrm{mS} \mathrm{m}^{-1}$ layer, $\sigma$ in all simulated soils was $\leq 100 \mathrm{mS} \mathrm{m}^{-1}$ (one of McNeill's [1980] criteria for the LIN conditions). A soil model in which the upper layer $\sigma$ was $0.1 \mathrm{mS} \mathrm{m}^{-1}$ and lower layer $\sigma$ was $0.2 \mathrm{mS} \mathrm{m}^{-1}$ is referred to as the [0.1/0.2] soil model. Induction numbers calculated from the results of simulations ranged from a minimum of about 0.01 for the [0.1/0.2] and [0.2/ $0.1]$ soil models to between 0.23 and 0.32 for the [100/ 200] and [200/100] soil models (Table 1). One conclusion of the LIN approximation is that vertical sensitivity, and therefore $d_{\mathrm{e}}$, does not depend on $\sigma_{\mathrm{a}}$. Results of simulations, however, indicate that instrument spatial sensitivity varies significantly with changes in $\sigma_{\mathrm{a}}$. This was true for Type 1 and 2 models for both homogeneous (e.g., the [0.1/0.2] and [100/99] soil models), as well as heterogeneous soil models. The general shape of all cumulative sensitivity distributions was similar to that predicted by the LIN approximation. In the more electrically resistive soils simulated, [0.1/0.2] and [0.2/0.1], CS values were close to those predicted by the LIN approximation (Fig. 4). In soils with higher $\sigma_{\mathrm{a}}$, however, the simulated 

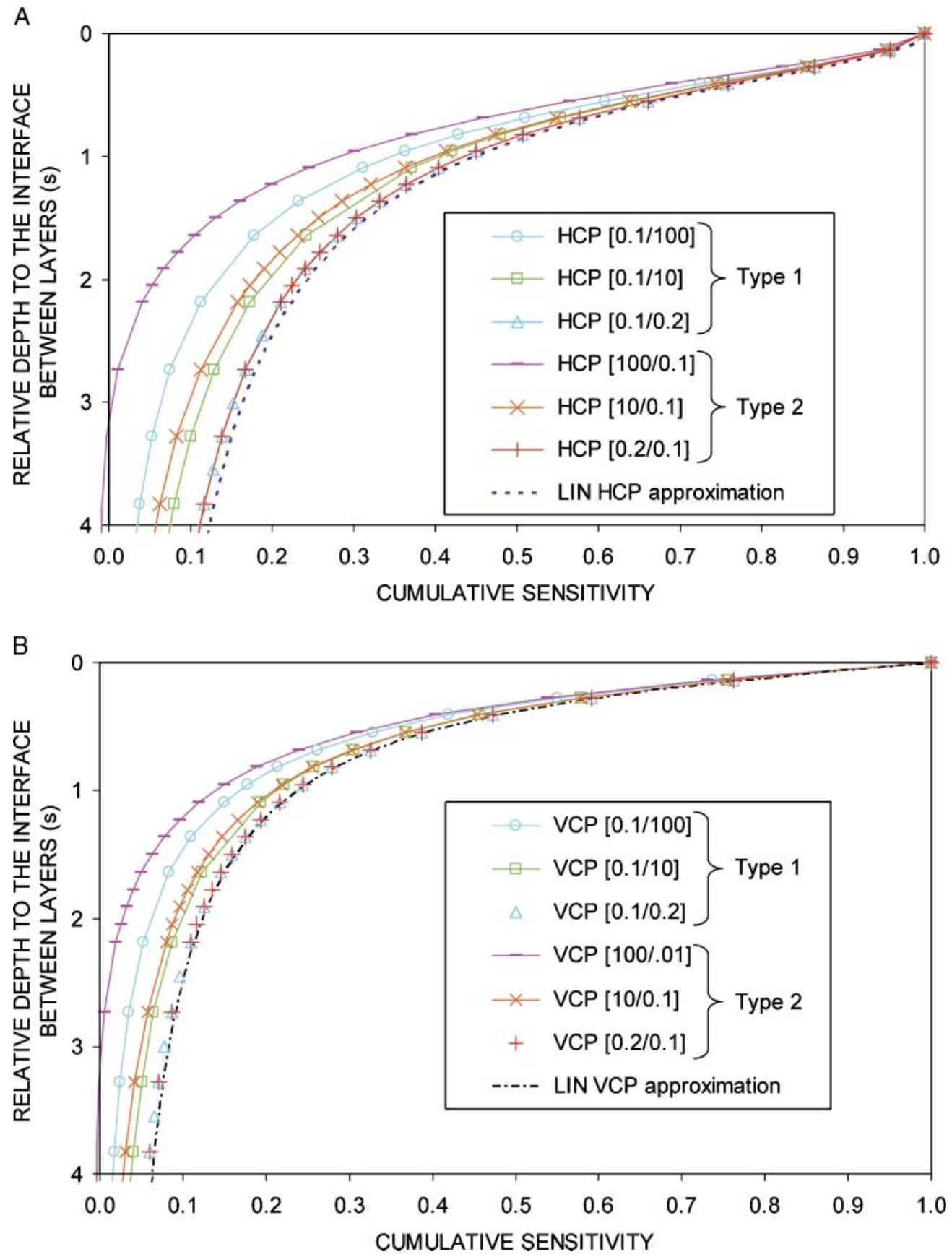

Fig. 4. Cumulative sensitivity for soil model Types 1 and 2 calculated from forward numerical simulation results compared with low-inductionnumber (LIN) approximation: (A) horizontal (HCP) and (B) vertical (VCP) coplanar coil orientation. Relative depth is depth divided by intercoil separation. The first number in brackets is the upper layer electrical conductivity, the second number is lower layer electrical conductivity, both in $\mathrm{mS} \mathrm{m}^{-1}$. In (B), to show underlying LIN VCP approximation curves, the lines for the VCP $[0.1 / 0.2]$ and $[0.2 / 0.1]$ soil models were removed.

CS values were at shallower depths than comparable LIN approximation values. For example, in Fig. 4A, the HCP LIN approximation predicts that a CS value of 0.2 will occur at a depth of about $2.5 s$. In contrast, the numerical approach predicts that as $\sigma$ increases, the CS depth decreases from just under $2.5 s$ for the $[0.2 / 0.1]$ and [0.1/0.2] soil models to $2.0 s, 1.8 s$, and $1.5 s$ for the [0.1/10], [10/0.1], and [0.1/100] soil models, respectively. The minimum CS depth was about $1.2 s$ for the [100/0.1] soil model. Although nearly all simulated $\sigma_{\mathrm{a}}$ values are within the LIN range indicated by McNeill (1980) and Frischknecht (1987), only simulations of the [0.1/0.2] and $[0.2 / 0.1]$ soil models $(\beta \sim 0.01)$ came close to the vertical sensitivity distribution predicted by the LIN approximation (Fig. 4). This indicates that only for electrically resistive soils do the LIN approximation and its predictions of the effective depth of exploration hold. Thus for many soils, depth to targets such as the water table or a particular soil horizon may be misjudged even at moderate values of $\sigma_{\mathrm{a}}$. For electrically conductive soils, 

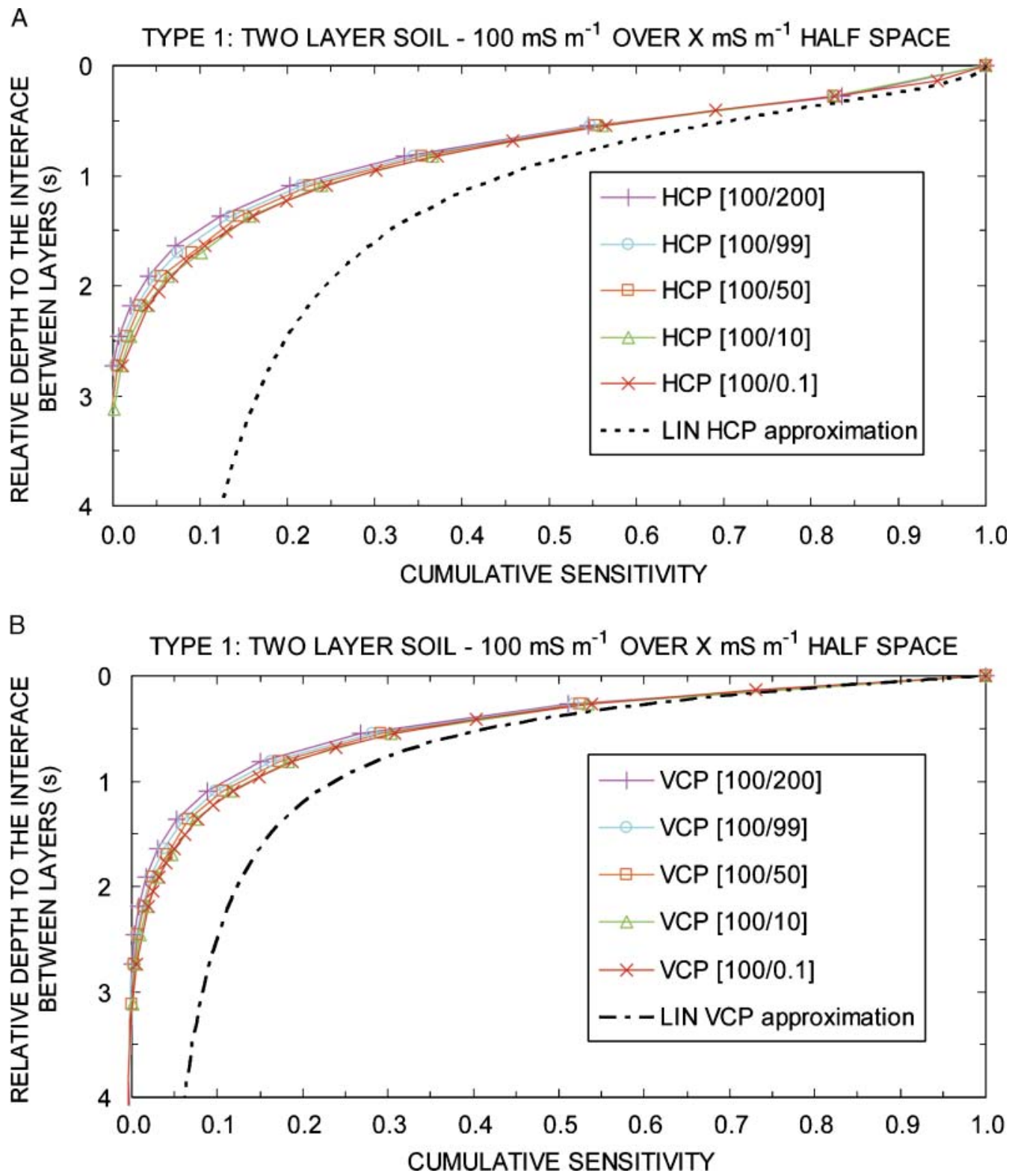

Fig. 5. Soil model Type 1 cumulative sensitivity using the higher electrical conductivities sometimes detected in environmental investigations. Comparison of results of forward numerical simulation with the low-induction-number (LIN) approximation: (A) horizontal (HCP) and (B) vertical (VCP) coplanar coil orientation. Relative depth is depth divided by intercoil separation. The first number in brackets is the upper layer electrical conductivity, the second number is that of the lower layer, both in $\mathbf{m S ~ m} \mathbf{~ m}^{-1}$.

for instance those that are clay rich, salty, or wet, such targets may be missed entirely.

There were a number of similarities and differences between the results of Type 1 and 2 soil-model simulations. In both model types, lower CS values occurred in soil with the higher upper-layer $\sigma$. For instance, in the [0.1/10] and [10/0.1] soil models, lower CS values occurred in the [10/ 0.1 ] soil model. This indicates that a LIN instrument would be less sensitive at depth to a soil with a [10/0.1] layering than to one with a [0.1/10] layering. Thus, it may be difficult to distinguish temporal or spatial variability of $\sigma_{\mathrm{a}}$, for example while tracking changes in salt or moisture content, below an electrically conductive surface layer. For a given $\sigma$ contrast, soil models with varied lower layer $\sigma$ (Type 1) were more similar to one another than soil models with varied upper layer $\sigma$ (Type 2). Deeper layers seemed to have less effect on VCP results than on HCP results, probably because the $\mathrm{VCP}$ orientation was more sensitive to shallow depths than the HCP orientation. For both orientations, Type 1 curves are more similar to one another than Type 2 curves, possibly because the source of variability, the top layer, is closer to the instrument. The last similarity to note is that, at any given depth, a nonlinear decrease in CS values occurred as the electrical conductivity of either layer increased. All these trends also occurred in higher conductivity soil models (Fig. 5 and 6).

McNeill's (1980) criterion for identifying $d_{\mathrm{e}}$ was $\mathrm{CS}=0.3$. Using this criterion for each numerical model, we determined $d_{\mathrm{e}}$ by locating the depth on each curve that corresponded to a CS value of 0.3 (Fig. 4-6). In 

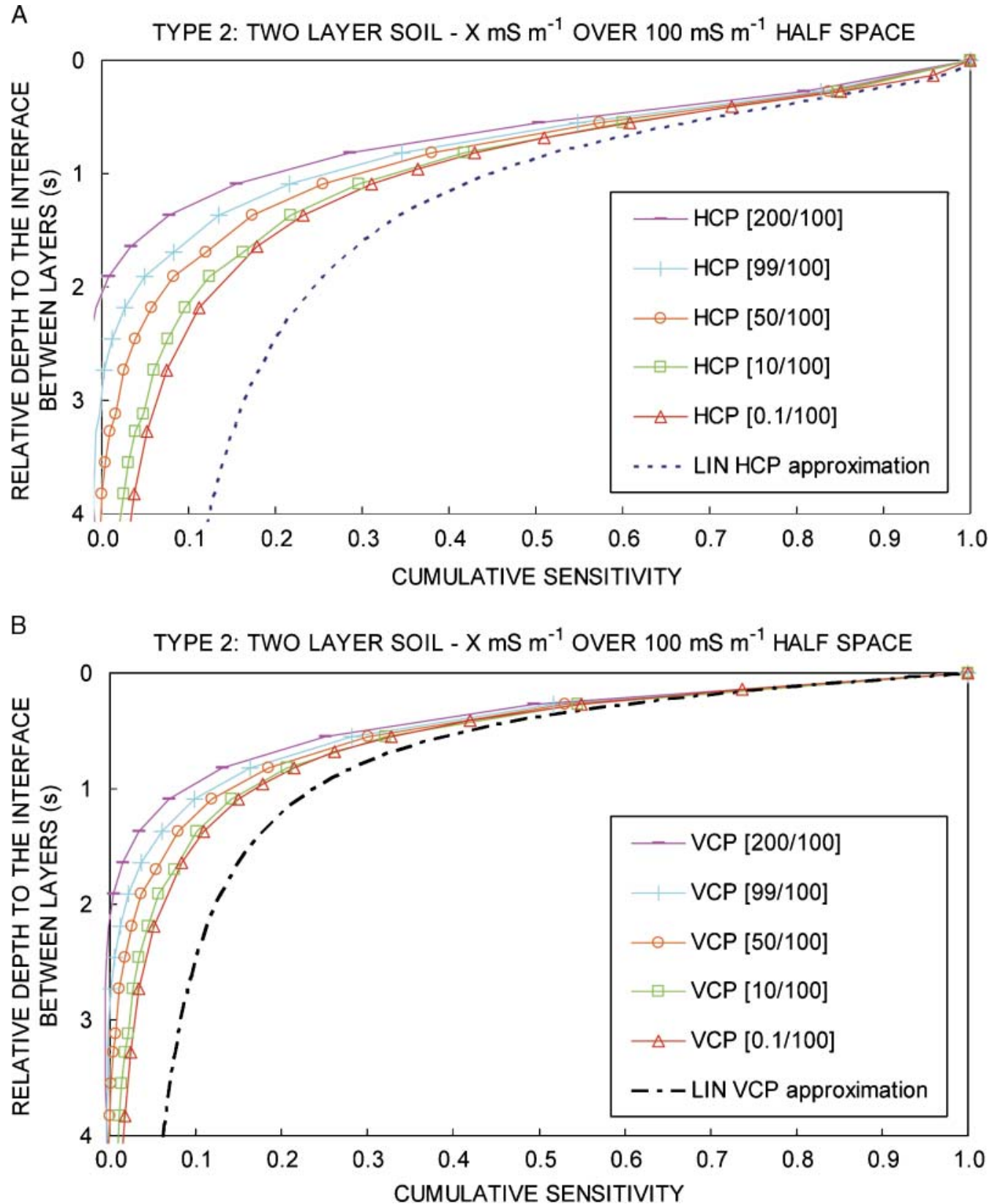

Fig. 6. Soil model Type 2 cumulative sensitivity using the higher electrical conductivities sometimes detected in environmental investigations. Comparison of results of forward numerical model simulation results with the low-induction-number (LIN) approximation: (A) horizontal (HCP) and (B) vertical (VCP) coplanar coil orientation. Relative depth is the depth divided by the intercoil separation. The first number in brackets is the upper layer electrical conductivity, the second number is the lower layer electrical conductivity, both in $\mathrm{mS} \mathrm{m}^{-1}$.

contrast to previous findings, $d_{\mathrm{e}}$ decreased with increasing $\sigma_{\mathrm{a}}$ (Fig. 7). This deviation from the LIN approximation was found in all soils simulated, including homogeneous and heterogeneous soils. The effect of increasing electrical conductivity on $d_{\mathrm{e}}$ was different for each coil orientation. For the VCP orientation, $d_{\mathrm{e}}$ for all numerical models tested ranged from 1.6s, the same depth as the LIN approximation, to about $0.8 s$ for the most electrically conductive soil. Using the VCP, LIN $d_{\mathrm{e}}$ could result in an overestimate of depth to soil-layer interfaces by as much as $100 \%$ of the actual $d_{\mathrm{e}}$. For the $\mathrm{HCP}$ orientation, $d_{\mathrm{e}}$ ranged from $0.76 \mathrm{~s}$ to $0.51 \mathrm{~s}$. Using the VCP, LIN $d_{\mathrm{e}}$ could result in an overestimate of as much as $50 \%$. Deviations of $10 \%$ or more from the LINpredicted depths occurred at $\sigma_{\mathrm{a}}$ values of $3 \mathrm{mS} \mathrm{m}^{-1}$ or greater. Given the range of near-surface $\sigma_{\mathrm{a}}$ in the continental USA (Keller and Frischknecht, 1966), reliance on the LIN assumptions at many field sites could lead to significant overestimates of $d_{\mathrm{e}}$ and poor estimates of layer thickness and composition, water content, or depth to interfaces such as the water table or changes in soil texture.

Considering the range of $\beta$, differences between the LIN and numerical approaches probably are caused by skin effect and scattering phenomena, which are not adequately represented by the LIN approximation. 


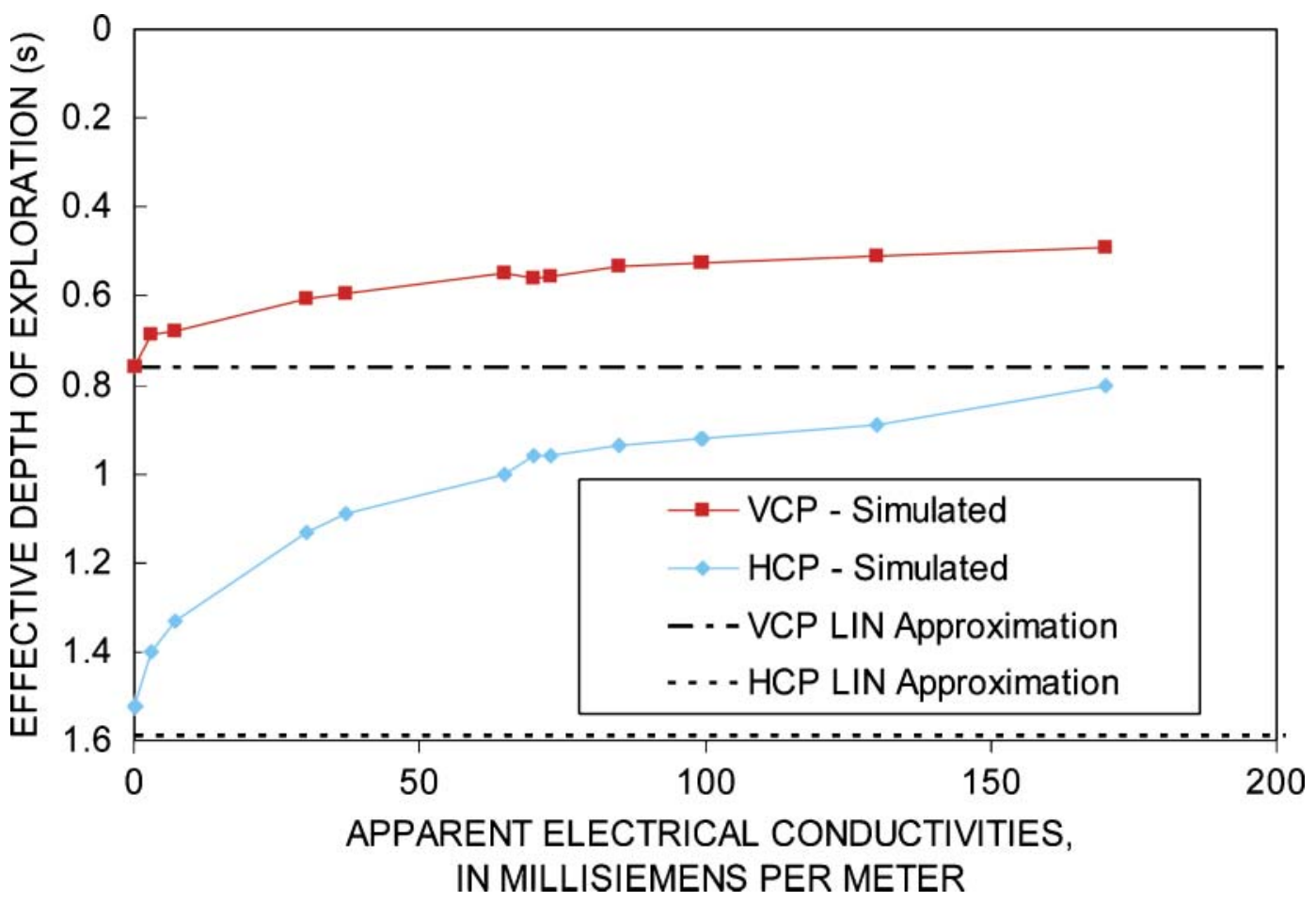

Fig. 7. Relation between apparent electrical conductivity and effective depth of exploration: comparison of simulated and low-induction-number (LIN) approximation results. VCP, vertical coplanar coil orientation; HCP, horizontal coplanar coil orientation. Effective depth of exploration is the depth divided by the intercoil separation.

Scattering can affect both primary and secondary magnetic fields. Both processes affect the cumulative secondary field sensed by the instruments at the surface. As a result, vertical variations in electrical conductivity will affect the results of field surveys by changing vertical spatial sensitivity. Temporal variations in water content, soil temperature, and salinity cause temporal variations in $\sigma_{\mathrm{a}}$, which may change $d_{\mathrm{e}}$. At any field site where physical, chemical, or hydraulic properties are spatially nonuniform, $d_{\mathrm{e}}$ is also likely to be nonuniform. Although the numerical data plotted in Fig. 7 will allow a better understanding of the behavior of $d_{\mathrm{e}}$ with changes in $\sigma_{\mathrm{a}}$, accurate determination of $d_{\mathrm{e}}$ may be difficult without the aid of numerical simulations.

\section{CONCLUSIONS}

Vertical spatial sensitivity and the effective depth of exploration of LIN FEM instruments in two-layer soils were studied using an industry-standard numerical model. The model simulation results were compared with interpretations based on the LIN asymptotic approximation developed previously. Clear differences were indicated between the predictions of the approximate and numerical approaches. In the numerical simulations, $d_{\mathrm{e}}$ decreased by up to $50 \%$ as the $\sigma$ of the soil increased when compared with the predictions of the LIN approximation. In the LIN approximation, vertical spatial sensitivity and $d_{\mathrm{e}}$ are independent of $\sigma_{\mathrm{a}}$. In contrast, the numerical models indicate that the magnitude and distribution of soil properties affecting electrical conductivity in the subsurface can significantly alter LIN instruments' vertical spatial sensitivity and consequently their $d_{\mathrm{e}}$. In electrically resistive soil models, where induction numbers were small $\left(\beta \leq 0.01\right.$ for a $0.1 \mathrm{mS} \mathrm{m}^{-1}$ upper layer over a $0.2 \mathrm{mS} \mathrm{m}^{-1}$ lower layer soil model), the vertical sensitivity distribution was similar to that predicted using the LIN asymptotic approximation. Under more electrically conductive conditions, even those that meet the LIN criteria of previous researchers, a lower CS than that predicted by the LIN approximation was observed at a given depth. The results indicate that in hydrologic and environmental investigations in any but the most electrically resistive environments, LIN FEM instrument sensitivity will probably be focused closer to the surface than predicted by the LIN approximation. This has important implications for studies of soil or geologic layering, water table detection, and studies of soil salinity, contaminant transport, and landfill delineation. In short, all studies that use LIN FEM instruments to study the vertical distribution of properties in the subsurface need to incorporate this information into project planning and data analysis. More heterogeneous subsurface conditions will benefit from numerical simulation of electromagnetic wave propagation to aid in the attribution of hydrologic properties, inferred from LIN FEM measurements, to appropriate subsurface locations. When access to software, time, or resources does not allow for numerical modeling, however, our plot of $d_{\mathrm{e}}$ vs. $\sigma_{\mathrm{a}}$ may be used to estimate the $d_{\mathrm{e}}$ based on reasonable estimates of the subsurface electrical conductivity.

\section{REFERENCES}

Anderson, W.L. 1984. Computation of Green's tensor integrals for three-dimensional electromagnetic problems using fast Hankel transforms. Geophysics 49:1754-1759. 
António, F., and L. Pacheco. 2002. Response to pumping of wells in sloping fault zone aquifers. J. Hydrol. 259:116-135.

Belluigi, A. 1949. Inductive coupling of a homogeneous ground with a vertical coil. Geophysics 14:501-507.

Benjoudi, H., P. Weng, R. Guérin, and J.F. Pastre. 2002. Riparian wetlands of the middle reach of the Seine River (France). Historical development, investigation and present hydrologic functioning: A case study. J. Hydrol. 263:131-155.

Frischknecht, F.C. 1987. Electromagnetic physical scale modeling. p. 365-441. In M.N. Nabighian, and J.D. Corbett (ed.) Electromagnetic methods in applied geophysics. Vol. 1. Soc. of Exploration Geophysicists, Tulsa, OK.

Gomez-Treviño, E., F.J. Esparza, and S. Méndez-Delgado. 2002. New theoretical and practical aspects of electromagnetic soundings at low induction numbers. Geophysics 67:1441-1451.

Kaufman, A.A., and G.V. Keller. 1983. Frequency and transient soundings. Elsevier, New York.

Keller, G.V., and F.C. Frischknecht. 1966. Electrical methods in geophysical prospecting. Pergamon Press, New York.

Lanz, E., D.E. Boerner, H. Maurer, and A. Green. 1998. Landfill delineation and characterization using electrical, electromagnetic and magnetic methods. J. Environ. Eng. Geophys. 3(4):185-196.

Lesch, S.M., J. Herrero, and J.D. Rhoades. 1998. Monitoring for temporal changes in soil salinity using electromagnetic induction techniques. Soil Sci. Soc. Am. J. 62:232-242.

Matias, M.S., M. Marques da Silva, P. Ferreira, and E. Ramalho. 1994. A geophysical and hydrogeological study of aquifers contamination by a landfill. Appl. Geophys. 32:155-162.

McNeill, J.D. 1980. Electromagnetic terrain conductivity measurement at low induction numbers. Tech. Note TN-6. Geonics Ltd., Mississauga, $\mathrm{ON}$.

Nyquist, J.E., and M.S. Blair. 1991. A geophysical tracking and data logging system. Description and case history. Geophysics 56 : 1114-1121.

Paine, J.G. 2003. Determining salinization extent, identifying salinity sources, and estimating chloride mass using surface, borehole, and airborne electromagnetic induction methods. Water Resour. Res. 39(3):1059, doi:10.1029/2001WR000710.

Pellerin, L., and P.E. Wannamaker. 2005. Multi-dimensional electromagnetic modeling and inversion with application to near-surface earth investigations. Comput. Electron. Agric. 46:71-102.

PetRos EiKon, Inc. 2004. EMIGMA. Release 7.7. PetRos EiKon, Inc., Concord, ON.
Salama, R.B., G. Bartle, P. Farrington, and V. Wilson. 1994. Basin geomorphological controls on the mechanism of recharge and discharge and its effect on salt storage and mobilization-Comparative study using geophysical surveys. J. Hydrol. 155:1-26.

Schneider, J.C., and S.E. Kruse. 2003. A comparison of controls on freshwater lens morphology of small carbonate and siliciclastic islands. Examples from barrier islands in Florida, USA. J. Hydrol. 284:253-269.

Sheets, K.R., and J.M.H. Hendrickx. 1995. Noninvasive soil water content measurement using electromagnetic induction. Water Resour. Res. 31:2401-2409.

Spies, B.R. 1989. Effective depth of exploration in electromagnetic sounding methods. Geophysics 54:872-888.

Stroh, J.C., S. Archer, J.A. Doolittle, and L. Wilding. 2001. Detection of edaphic discontinuities with ground-penetrating radar and electromagnetic induction. Landsc. Ecol. 16:377-390.

Sudduth, K.A., N.R. Kitchen, W.J. Wiebold, W.D. Batchelor, G.A Bollero, D.G. Bullock, D.E. Claye, H.L. Palm, F.J. Pierce, R.T. Schuler, and K.D. Thelen. 2005. Relating apparent electrical conductivity to soil properties across the north-central plains. Comput. Electron. Agric. 46:263-283.

Tabbagh, A. 1985. The response of a three-dimensional magnetic and conductive body in shallow depth electromagnetic prospecting. Geophys. J. R. Astron. Soc. 81:215-230.

Telford, W.M., L.P. Geldart, and R.E. Sheriff. 1990. Applied geophysics. Cambridge Univ. Press, Cambridge, UK.

Triantafilis, J., and S.M. Lesch. 2005. Mapping clay content variation using electromagnetic induction techniques. Comput. Electron. Agric. 46:203-237.

Wait, J.R. 1955. Mutual electromagnetic coupling of loops over a homogeneous ground. Geophysics 20:630-637.

Wait, J.R. 1962. A note on the electromagnetic response of a stratified earth. Geophysics 27:382-385.

Wannamaker, P.E., G.W. Hohmann, and W.A. SanFilipo. 1984 Electromagnetic modeling of three-dimensional bodies in layered earths using integral equations. Geophysics 49:60-74.

Ward, S.H., and G.W. Hohmann. 1988. Electromagnetic theory for geophysical applications. p. 131-312. In M.N. Nabighian (ed.) Electromagnetic methods in applied geophysics. Vol. 1. Soc. of Exploration Geophysicists, Tulsa, OK.

Yoder, R.E., R.S. Freeland, J.T. Ammons, and L.L. Leonard. 2001. Mapping agricultural fields with GPR and EMI to identify offsite movement of agrochemicals. J. Appl. Geophys. 47:251-259. 